\title{
Comparison of Release Rates Derived from Cutaneous Versus Film-Forming Solution Containing Terbinafine: Approach Towards Qualification and Validation of In Vitro Parameters
}

Yogesh Upadhyay ${ }^{1 *}$, Abhishek Kumar Singh ${ }^{1}$, Dr. Sanjeev Mishra ${ }^{1}$, Sanjay Jagannath Gurule ${ }^{1}$, Arshad H. Khuroo' ${ }^{1}$, Soumya Verma², Neeta Tiwari², and Simrata Bedi²

${ }^{1}$ Department of Clinical Pharmacology and Pharmacokinetics, Sun Pharmaceutical Industries Ltd., Gurugram, Haryana, India.

${ }^{2}$ Department of Formulation Research Development-Orals, Sun Pharmaceutical Industries Ltd., Gurugram, Haryana, India.

e-mail: Yogesh.Upadhyay@sunpharma.com

\begin{abstract}
Excipients play a very important role in the release pattern of an active pharmaceutical ingredient from topical semisolid dosages forms, and their physical and chemical properties can influence the release. The aim of this paper was to provide a validated, sensitive, and reproducible method to assess the in vitro release rate of an antifungal drug (Terbinafine) from controlled drug delivery systems (cutaneous and film-forming solution) and to prove product sameness. The samples obtained from in vitro testing were analyzed through a high performance liquid chromatographic (HPLC) system coupled with UV spectrometer at a wavelength of $283 \mathrm{~nm}$. A Franz diffusion cell (FDC) system was used for the dissolution test. We recorded the drug release from the formulation for 6 hours. The release rate obtained from cutaneous and filmforming solutions were compared statistically to depict the sameness. The results met the relevant acceptance criteria (i.e., $90 \%$ confidence interval, falling within the limits of 75-133\%) as defined in the scale-up and post-approval changes (SUPAC-SS) guidance. The obtained results confirm the competence of the IVRT method for the assessment of product sameness.
\end{abstract}

KEYWORDS: In vitro release testing, Franz diffusion cell, apparatus qualification, terbinafine, dissolution

\section{INTRODUCTION}

T he allylamine class of antifungals is highly lipophilic. They inhibit fungal growth by disrupting sterol biosynthesis. It abrogates the formation of ergosterol by inhibiting squalene epoxidase, the catalytic enzyme responsible for converting squalene to 2, 3-oxidosqualene (an ergosterol precursor). These drugs are indicated to treat fungal skin and nail infections caused by Trichophyton species, Microsporum canis, Epidermophyton floccosum, and Tinea species. They also treat yeast infections of the skin caused by Candida species (1).

Recently, there has been an increased interest in drug administration via the skin (topical delivery) for both local therapeutic effects and for systemic delivery (transdermal delivery). We can measure the permeation of chemicals through the skin by in vivo and in vitro techniques. Frequently, this has been done by in vitro techniques because of the simplicity of the experimental conditions. The in vitro release test (IVRT) is used to monitor the release and diffusion of drug products from semisolid dosage forms and has long been considered as a valuable tool in formulation development. IVRT has also been used to screen formulations to select promising candidates and, importantly, is accepted with the purpose of obtaining a waiver of bioequivalence studies following post-approval changes to a product (2).

Advantages of the IVRT are quality control of drug formulations, prediction of their in vivo behavior, estimation and assurance of desired formulation design, quality of formulation, and assessment of product sameness after post-approval changes. IVRT has been essential in determining product sameness, particularly during any change in formulation, excipient, 
or manufacturing process/site. Besides its use in quality control, IVRT can also optimize formulation during the early stages of development. IVRT is a cost-effective alternative for providing some predictive estimates regarding the in vivo performance of a drug product.

The development of controlled drug delivery systems has generated considerable interest in pharmaceutical science in recent years. In particular, the transdermal drug delivery has attracted researchers with multiple approaches because multiple dosing or insufficient drug delivery often results in low therapeutic effects. Among these techniques, films (patches) and gels have been extensively designed for skin diseases or wound care in the past decades. These dosage forms can also contain drugs for therapeutic applications. Fortunately, the combined advantages of both film and hydrogels were found in filmforming solutions (FFSs) for transforming the drug via thin film. In fact, FFSs contain three key components, i.e., the drug, film-forming polymer, and solvent(s). Upon coming in contact with the target site (usually skin), the solvent will evaporate to form a film-loading drug (3).

The FFS technique of drug delivery is an effective and novel approach for the delivery of drug in skin. This technique was developed and categorized in agreement with their mechanical properties and water vapor permeability. For making FFSs, the drug and film-forming excipient are dissolved/dispersed in a volatile solvent(s). The liquid state of the FFS depends on the solubility of drug/excipient or dispersions of encapsulated drug micro particles/nanoparticles in solvents. After coming in contact with the skin, the solvents evaporate and form a film with excipient. With FFSs, the polymer can have a tight contact via molecular interactions to build an even film or smooth film, thus facilitating prolong release (3).

The current research work aims to qualify and validate the IVRT and high performance liquid chromatography (HPLC) method and to specify the procedure for providing the product sameness by comparing the release rate of terbinafine (TBF, 1\%) from its cutaneous and film-forming solutions. The selection of receptor medium for estimating the release rate of TBF through synthetic membrane was challenging, as TBF degraded rapidly in phosphate buffered saline/aqueous media. However, in organic/ organic-aqueous media, over $30 \%$ of cumulative release was observed, showing deviation from Higuchi theory (2, 4). Moreover, the coefficient of determination $\left(R^{2}\right)$ was less than $0.90 \%$ and inter cell precision greater than $15 \%$. These issues were resolved by lowering the $\mathrm{pH}$ of receptor medium to approximately 5.5 using ascorbic acid in an optimized quantity, which prevented the degradation of
TBF. Moreover, by lowering the $\mathrm{pH}$ of receptor solution, precise and accurate results were obtained, and the sink condition was maintained throughout the experimental duration.

An extensive literature search revealed that no IVRT method has been published for estimating the release rate of TBF from cutaneous and film-forming solutions. We found some analytical methods for determination of TBF via UV spectrophotography or chromatographically (5).

\section{MATERIALS AND METHODS \\ Chemicals and Reagents}

Terbinafine hydrochloride (working standard) was procured from Sun Pharmaceutical Industries LTD., Baddi, India. Acetonitrile and methanol (HPLC Grade) were purchased from Sigma Aldrich Chemical Pvt. Ltd., Bengaluru, India. Trifluoroacetic acid and ascorbic acid (analytical grade) were purchased from Thermo Fisher Scientific, Haryana, India. Phosphate buffered saline was purchased from Sisco Research Laboratories Pvt. Ltd., Mumbai, India.

\section{Equipment}

IVRT experiments were performed in a Franz diffusion cell (FDC) from PermeGear, Pennsylvania, USA. Synthetic membranes for IVRT experiments, i.e., Ultipor N66, Nylon 6,6 $(0.2 \mu \mathrm{m} \times 25 \mathrm{~mm}$, Lot No. IN15000705; $0.2 \mu \mathrm{m} \times 25$ $\mathrm{mm}$, Lot No. IN12000094), Tuffryn HT-200, (0.2 $\mu \mathrm{m} \times$ $25 \mathrm{~mm}$, Lot No. T30120), and Supor $200(0.2 \mu \mathrm{m} \times 25$ $\mathrm{mm}$, Lot No. IN14000045) were purchased from Pall Life Sciences, Mumbai, India. For temperature monitoring of synthetic from Metravi, MT-4, West Bengal, India was used. For quantification of IVRT samples, HPLC system coupled with UV detector from Shimadzu, Mumbai, India was used along with Analyst 1.6.3 software for data processing. All statistical calculations were done through Microsoft Excel 2013.

\section{Drug Products}

Terbinafine 1\% cutaneous solution (Lamisil Once, batch no. 8U6G, Galaxo SmithKline, Brentford, UK) was used as reference formulation and terbinafine 1\% FFS (batch no. SMV(7151)080, Sun Pharmaceutical Industries Ltd., Gurugram, India) was used as test formulation. Additionally, for IVRT selectivity, specificity, and sensitivity experiments, two more test formulations were used, i.e. terbinafine $0.5 \%$ and $2.0 \%$ FFS (batch nos. SMV(7151)088, SMV(7151)090, Sun Pharmaceutical Industries Ltd).

\section{HPLC-UV Method Validation}

RP-HPLC method validation was performed in the range of 0.501 to $121.000 \mu \mathrm{g} / \mathrm{mL}$ at $283 \mathrm{~nm}$ using Zorbax Eclipse

Dissolution 
XDB-C8 $(150 \times 4.6,5 \mu)$ column from Waters, Hyderabad, India, with the mobile phase comprising of solution 1 (acetonitrile:methanol; 50:50 v/v) in combination with solution $2(0.3 \%$ trifluoroacetic acid in water, $v / v)$ in the ratio of $45: 55 \mathrm{v} / \mathrm{v}$, with a $0.700 \mathrm{~mL} / \mathrm{min}$ flow rate. The injection volume was kept as $10 \mu \mathrm{L}$ and the column oven temperature was set at $45{ }^{\circ} \mathrm{C}$. We prepared separate stock solutions of TBF in methanol to generate a calibration curve $(1 \mathrm{mg} / \mathrm{mL})$ and for preparing quality control samples. The stock solution was further diluted with mobile phase to yield concentrations of $0.501,1.210$, $6.050,12.100,24.200,48.400,96.800$, and $121.000 \mu \mathrm{g} /$ $\mathrm{mL}$. In each analytical run of IVRT samples, a set of eight calibration standards along with one blank were injected. The regression curve was established from all calibration standards. Additionally, at least three levels of QC samples (in the concentrations of $1.253,48.200,96.400 \mu \mathrm{g} / \mathrm{mL}$, i.e., low, middle, and high quality control respectively) were interspersed with each IVRT run.

\section{IVRT Method}

IVRT was performed using an FDC system, having receptor chamber volume with the capacity of $20 \mathrm{~mL}$. All components of the FDC system such as donor chamber, receptor chamber, clamp, magnetic stirrer, and synthetic membranes were arranged. A magnetic stirrer was placed inside the receptor chamber of FDC. The receptor chamber was filled with receptor solution, i.e., 10x phosphate buffered saline: water, 10:90 v/v containing $7 \%$ Ascorbic acid w/v. Membrane was mounted over the receptor chamber until it touched the joint surface between the receptor chamber and the donor chamber. The donor chamber was placed on top of the membrane properly aligning with FDC assembly. A clamp was affixed to stabilize and secure the joint between the donor chamber and the receptor chamber. The underside of each membrane was checked for air bubbles, if any, then it was tilted to remove the air bubbles. The FDC assembly was placed within a cell holder and a port of the water jacket was connected to the tubing of the re-circulator rack. The heating circulator bath was turned on, and the temperature of the water tank was set appropriately to maintain membrane at about $32 \pm 1^{\circ} \mathrm{C}$. Magnetic stirrer was turned on (with a fixed speed of approximately $560 \mathrm{rpm}$ ) throughout the test. The membrane was kept in equilibration for $30 \mathrm{~min}$. The temperature on the membrane surface was measured using a calibrated infrared thermometer. Prior to application of formulation on the membrane surface, pre-dose samples (300 $\mu \mathrm{L})$ were collected from the centre of the receptor chamber from each FDC and transferred into sample collection vials. Stock receptor solution was used for replenishing the receptor chamber after each sampling. The components of the FDC are shown in Figure 1.

\section{IVRT Apparatus and Laboratory Qualification}

The IVRT apparatus was qualified with all critical parameters of the FDC. These parameters include assessment of the capacity of the receptor chamber, diameter of FDC, temperature on membrane surface and in receptor solution, the stirring speed, dispensing volume, and environment conditions (7). All these parameters are measured by length, weight, and temperature measurement. Results are shown in Table 1.

The laboratory qualification was performed by assessing the release rates of TBF reference formulations with the developed and validated IVRT and HPLC-UV method. Release rates of reference formulation were evaluated on

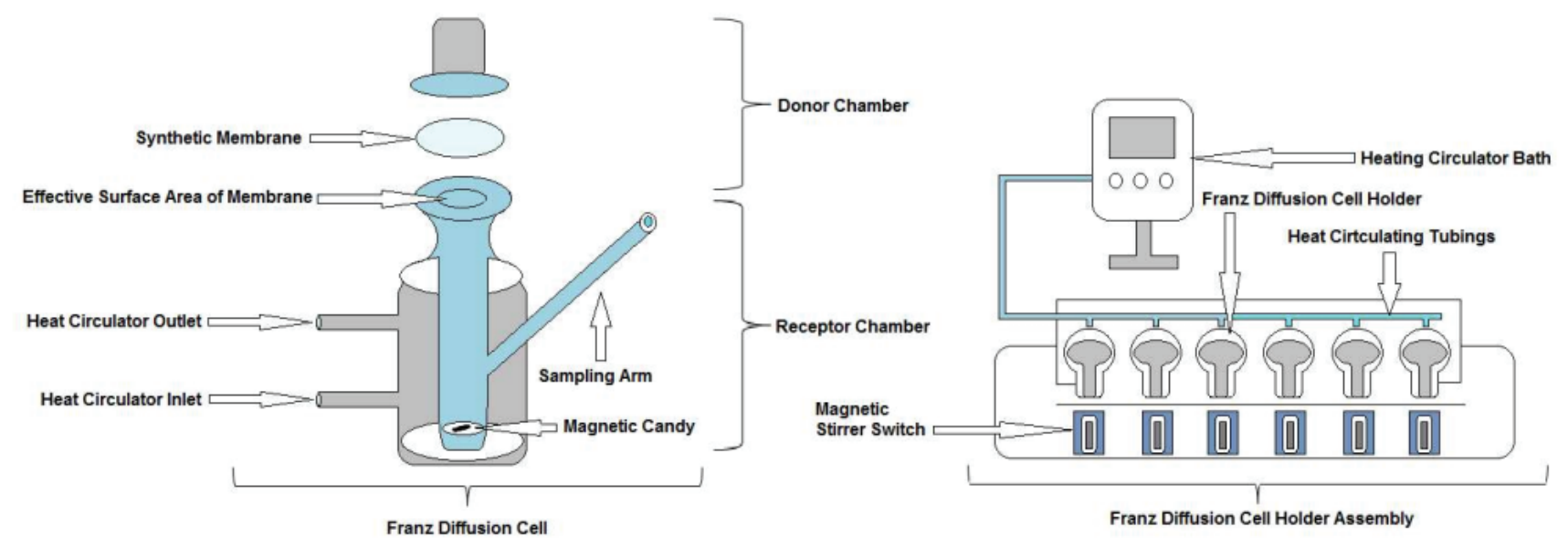

Figure 1. Components of a Franz diffusion cell system. 
two different days ( $n=6$ FDCs per day). Reproducibility, intra- and inter-run variability was determined in term of percent coefficient of variation (\%CV). The $\% \mathrm{CV}$ should be $<15 \%$. The intra-run \%CV for the first and second IVRT runs were calculated as $8.98 \%$ and $4.01 \%$, respectively; inter-run \%CV ( $n=12$ FDCs) was calculated as $3.82 \%$. The product sameness test was based on computation of $90 \%$ confidence interval $(\mathrm{Cl})$ as per scale-up and postapproval changes (SUPAC SS) guidance (6). Individual test/reference (T/R) ratios were calculated in percentage terms by considering the first day run as reference and the second day run as test. The $90 \% \mathrm{Cl}$ was subsequently determined from the ordered T/R ratios, where the 8th and 29th ordered individual T/R ratios are the lower and the upper limits, respectively (6). For equivalence, the calculated $90 \% \mathrm{Cl}$ should be within the range of $75-133 \%$. The lower limit and upper limit of $90 \% \mathrm{Cl}$ was calculated as $99.09 \%$ and $120.08 \%$, respectively, and the same was falling in the acceptable range.

Table 1. Results of Apparatus Qualification Test

\begin{tabular}{|c|c|c|c|}
\hline Parameter & $\begin{array}{c}\text { Acceptance } \\
\text { Criteria }\end{array}$ & Result & Acceptable \\
\hline FDC capacity $(\mathrm{mL})$ & $20 \pm 1.0$ & $20 \pm 0.1$ & YES \\
\hline Orifice diameter $(\mathrm{mm})$ & $15 \pm 0.75$ & $15 \pm 0.2$ & YES \\
\hline $\begin{array}{c}\text { Temperature of } \\
\text { receptor solution }\left({ }^{\circ} \mathrm{C}\right)\end{array}$ & $32 \pm 1$ & $32 \pm 0.4$ & YES \\
\hline $\begin{array}{c}\text { Temperature on } \\
\text { membrane surface }\left({ }^{\circ} \mathrm{C}\right)\end{array}$ & $32 \pm 1$ & $32 \pm 0.5$ & YES \\
\hline $\begin{array}{c}\text { Speed of magnetic } \\
\text { stirrer }(\text { rpm })\end{array}$ & $600 \pm 60$ & $565 \pm 5$ & YES \\
\hline $\begin{array}{c}\text { Dispensed sampling } \\
\text { volume }(\mu \mathrm{L})\end{array}$ & $300 \pm 9$ & $302 \pm 6$ & YES \\
\hline
\end{tabular}

Values are presented as mean $\pm S D(n=6)$.

FDC: Franz diffusion cell; SD: standard deviation.

\section{Selection of Receptor Solution}

The receptor solution should be selected to maintain the sink conditions throughout the IVRT experiment. In this method, receptor solution was selected based on solubility, reproducibility of results, and $R^{2}$ (which needs to be $\geq 0.90$ ). Solubility of TBF in receptor solution was verified by dissolving $1 \mathrm{mg}$ of TBF in $1 \mathrm{~mL}$ of receptor solution.

\section{Selection of Membrane Using Membrane Binding Test Method}

This test was performed to determine the suitability of a synthetic membrane in providing inertness for the diffusion of drug across it from a semisolid dosage form. Supor-200, Ultipor, and Tuffryn HT-200 synthetic membranes were used to evaluate membrane binding test. These membranes were dipped in a known concentration of TBF prepared in a receptor solution for at least 6 hours. After the storage period, membranes dipped in TBF solutions were injected and compared with the peak area response got from controlled stock solution.

\section{Formulation Application and Sample Collection}

Formulation was applied on the membrane surface of each FDC using a syringe containing approximately 800 $\mu \mathrm{L}$ and was spread evenly. The donor chamber was occluded. At pre-set sampling time points (predose, 0.5, $1,2,3,4,5$, and 6 hours) $0.300 \mathrm{~mL}$ of receptor solution was removed from the center of the receptor chamber and transferred into HPLC glass vials. The receptor chamber was replenished with pre-warmed receptor solution.

\section{Selection of Sampling Time Points}

Sampling time points were as follows: predose, $0.5,1,2$, $3,4,5$, and 6 hours. The sampling time of the experiment for 6 hours was found sufficient to discriminate between different strengths (i.e., $0.5 \%, 1.0 \%$, and $2.0 \% \mathrm{w} / \mathrm{w}$ ) of TBF film forming solution.

\section{IVRT Sample Quantification}

After the completion of the experiment, the concentration at each sampling time point was obtained through HPLC analysis. The amount released at each sampling time point $\left(\mu \mathrm{g} / \mathrm{cm}^{2}\right)$ was calculated as the cumulative concentration obtained at each sampling time point $(\mu \mathrm{g} / \mathrm{mL}) \times$ volume of FDC $(\mathrm{mL})(20 \mathrm{~mL}) \times$ amount of sample removed from FDC at each sampling time $(\mathrm{mL})$, all divided by effective surface area of membrane (surface area of orifice $=1.77 \mathrm{~cm}^{2}$ ). Amount of sample removed from FDC in each sampling time $(\mathrm{mL})$ was summed up for the next sample to calculate the cumulative amount removed in previous sampling.

The in vitro release rate calculated by plotting cumulative amount of drug release per unit area $\left(\mu \mathrm{g} / \mathrm{cm}^{2}\right)$ against time $\left(h^{1 / 2}\right)$ yields a straight line, the slope of which gives release rate. The percent cumulative drug release was also calculated by dividing the cumulative amount release $(\mu \mathrm{g})$ by the amount of formulation applied.

\section{Statistical Comparison Between Reference and Test Formulations}

Comparison of in vitro release rates was carried out as per the mentioned method in SUPAC-SS guideline. Six slopes (release rates) were obtained from each test and reference formulations. From these slopes, a total of 36 individual $T / R$ ratios (slope of $T$ divided by $R$ ) were calculated in terms of percentages. These computed $T / R$ ratios were ranked from lowest to highest. The 8th and 29th ranked individual ratios are the lower and upper limit, respectively, of the $90 \% \mathrm{Cl}$ for the ratio of the in 
vitro release rate (slope) for T over $\mathrm{R}$. The $90 \% \mathrm{Cl}$ should fall between $75 \%$ to $133 \%$ (6).

\section{RESULTS AND DISCUSSION \\ HPLC Method Validation \\ Linearity and Range}

The linearity of the method was determined by analysis of standard plots associated with an eight-point standard calibration curve. The calibration curve was linear $(y=$ $21600 x+314$ ) from $0.501 \mu \mathrm{g} / \mathrm{mL}$ [limit of quantitation (LOQ)] to $121.000 \mu \mathrm{g} / \mathrm{mL}$ [upper limit of quantitation (ULOQ)] with correlation coefficient $(r)$ as 1.000 . Back-calculations were performed from that curve to determine concentrations of TBF in unknown samples.

\section{Selectivity Using Synthetic Membrane and Placebo}

For the HPLC method validation, the synthetic membrane (Ultipor N66) was dipped in the receptor solution for 6 hours. Similarly, $800 \mu \mathrm{L}$ of placebo was added to $20 \mathrm{~mL}$ of receptor solution, vortexed and kept in bench for 6 hours (covering the entire duration of the experiment). The same was done in triplicate. After analyzing the selectivity samples, the peak area response obtained at RT of analyte was evaluated. As a result, no significant interference was observed at RT of the analyte in selectivity blank samples. This indicates that the analytical method is selective for analysis of TBF from its film forming solution.

\section{Precision and Accuracy}

Precision of method is degree of reproducibility and accuracy is degree of exactness. Precision is denoted as $\%$ coefficient of variation, and accuracy is denoted as $\%$ deviation at each concentration level from nominal concentrations. Here, we have assessed within and between batch precision and accuracy. Within batch precision and accuracy was assessed by analyzing six replicated of quality control (QC) samples, on the same day at three levels of QC (i.e., 1.494, 48.184, and $96.368 \mu \mathrm{g} /$ $\mathrm{mL}$ for low, medium, and high QC, respectively) prepared in receptor solution. The between batch precision and accuracy was assessed by analyzing 18 replicates of QC samples, at each above-mentioned QC level, prepared in receptor solution thorough three precision and accuracy batches ran on two sequential validation days.

The deviation at each $\mathrm{QC}$ level from the nominal concentration was found to be $91.00-97.01 \%$ for within batch and $95.89-98.16 \%$ for between batch accuracy. Similarly, the mean precision was found to be $0.14-1.28 \%$ for within batch and 1.03-1.20\% for between batch precision.

\section{IVRT METHOD VALIDATION}

\section{Solubility of Drug in Receptor Solution}

Solubility of TBF in receptor solution, i.e., 10x phosphate buffered saline (water, 10:90 v/v containing 7\% ascorbic acid $w / v)$ was found to be excellent. The solubility of 1 $\mathrm{mg}$ of TBF in $1 \mathrm{~mL}$ of receptor solution was calculated as $762.675 \mu \mathrm{g} / \mathrm{mL}$ (calculated theoretical yield was $763.267 \mu \mathrm{g} / \mathrm{mL}$ ), and $99.92 \%$ of TBF was calculated as recovery. With this receptor solution, sink condition was maintained, release rates were reproducible and consistent $R^{2}$ was observed throughout the experiments.

\section{Selection of Synthetic Membrane}

Among the screened membranes, Supor 200 membrane and Tuffryn HT-200 membrane showed significant binding (7.82\% and $8.83 \%$ respectively) of TBF on the membrane whereas Ultipor $\mathrm{N} 66$ membrane provided the least binding (1.86\%). The higher recovery (98.14\%) showed that Ultipor $\mathrm{N} 66$ membrane provided more inertness in the diffusion of TBF from formulation across it and is therefore more appropriate for IVRT experiments.

\section{Sensitivity, Specificity, and Selectivity}

Sensitivity, specificity, and selectivity of this IVRT method was evaluated by investigating the rate of release of TBF from three test FFSs containing $0.5 \%, 1.0 \%$, and $2.0 \%$. Sensitivity of the IVRT method showed that it can successfully distinguish the three products as the mean release rate increases with increasing the TBF concentration, i.e., $142.22 \mu \mathrm{g} / \mathrm{cm}^{2} / \mathrm{h}^{1 / 2}$ for $0.5 \%, 349.53$ $\mathrm{\mu g} / \mathrm{cm}^{2} / \mathrm{h}^{1 / 2}$ for $1.0 \%$, and $876.30 \mu \mathrm{g} / \mathrm{cm}^{2} / \mathrm{h}^{1 / 2}$ for $2.0 \%$ (Fig. 2).

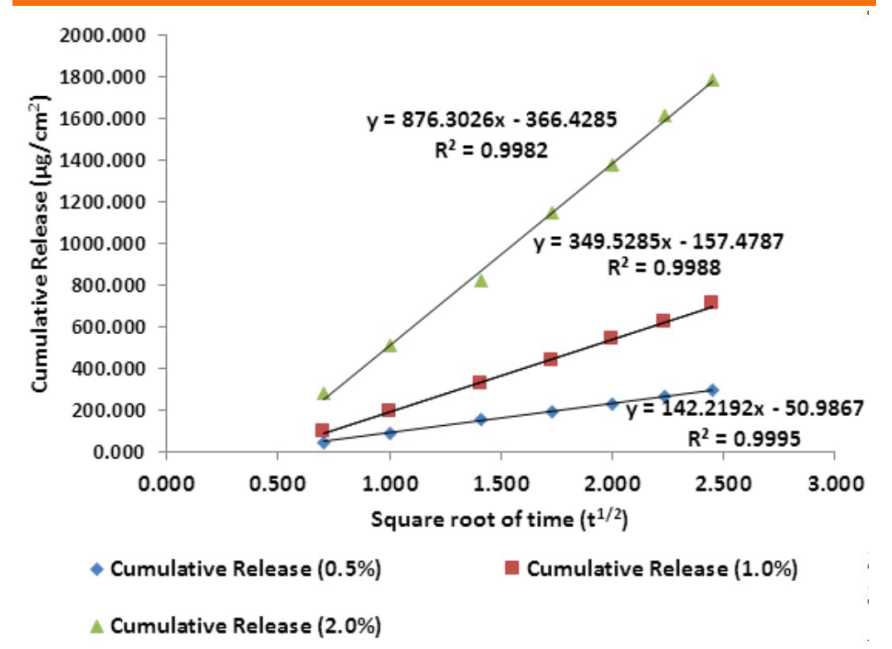

Figure 2. Cumulative release of terbinafine from different dosage strengths $(0.5 \%, 1.0 \%, 2.0 \% \mathrm{w} / \mathrm{w})$, showing sensitivity of method.

A linear regression model was used with the release rate as a dependent variable and TBF concentration of the respective test product as an independent variable to test 
for specificity. The results provided evidence of a linear $\left(R^{2}=0.9478\right)$, proportional relationship between TBF concentration in FFS and the resulting TBF release rates (Fig. 3).

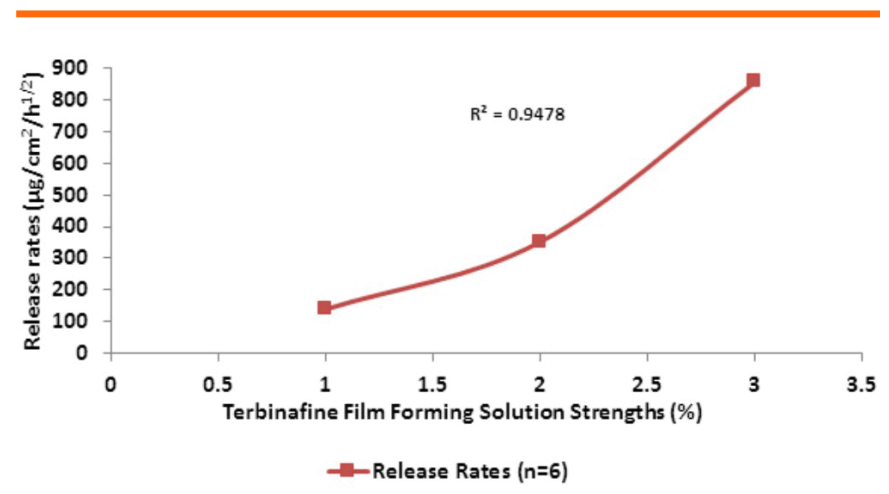

Figure 3. Coefficient of determination $\left(R^{2}\right)$ obtained from dissolution profile of terbinafine $(0.5 \%, 1.0 \%, 2.0 \%)$, showing specificity of the method.

To assess selectivity, the IVRT method should be able to accurately identify in-equivalent product performance. The in-equivalence of the product performance was tested by pair-wise comparisons of the $1.0 \%$ TBF FFS with either of the $0.5 \%$ or $2.0 \%$ TBF FFSs. The pre-determined criterion for equivalence is the calculated $90 \% \mathrm{Cl}$, i.e., $8^{\text {th }}$ and $29^{\text {th }}$ rank order should be within the range of 75$133 \%$. Here, the computed $8^{\text {th }}$ and $29^{\text {th }}$ rank order is not within the acceptable range (Tables 2 and 3); hence, the in-equivalence of these products was confirmed.

\section{Recovery}

Recoveries were evaluated in three runs of IVRT. For each run, six FDCs were used, and reference formulation was applied. Recoveries were calculated for three runs and found to be $21.13 \%, 20.70 \%$, and $21.45 \%$. The calculated recoveries were less than $30.00 \%$, with an acceptable linearity of TBF release rates throughout the duration; hence, the extent of TBF dose depletion was considered acceptable.

\section{Comparison of Release Rates: Cutaneous Solution Versus Film-Forming Solution}

Release rates were calculated for both formulations and are shown in Figure 4. $R^{2}$ was found to be greater than 0.90 which showed that the rate of drug release from the formulation was consistent over the period of time. The $\% \mathrm{CV}$ for the release rate was also calculated between each cell (within same day) and found to be less than $15 \%$, which shows low intra-cell variation and reproducibility of the method (Table 3). All the above factors indicated that this developed and validated IVRT method follows Higuchi theory $(2,4)$.

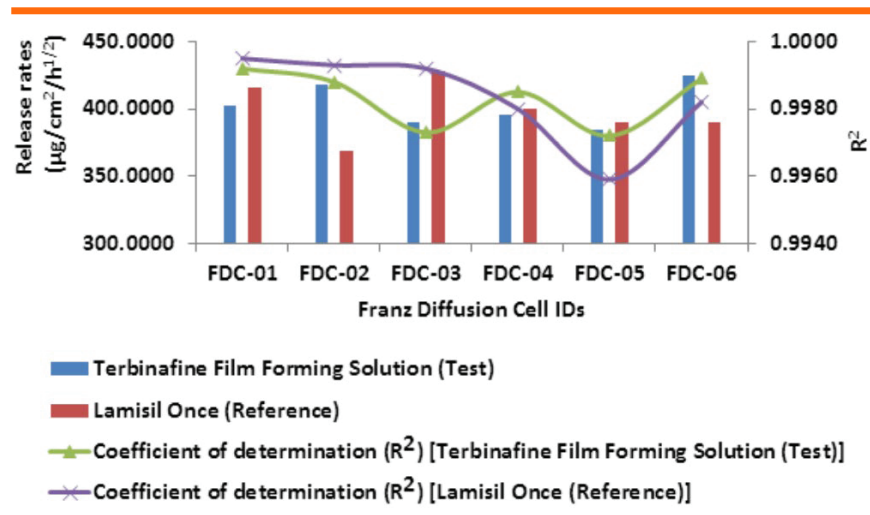

Figure 4. Release rates and coefficient of determination (R2) obtained from reference and test formulations containing $1 \%$ terbinafine.

\begin{tabular}{|c|c|c|c|c|c|c|c|}
\hline \multirow{3}{*}{ Test Release Rate (Slope) } & \multicolumn{7}{|c|}{ Reference Release Rate (Slope) } \\
\hline & & 415.3325 & 368.6100 & 427.7148 & 399.5709 & 390.1243 & 389.8974 \\
\hline & \multicolumn{7}{|c|}{ T/R Ratios (\%) } \\
\hline 149.1261 & \multirow{6}{*}{ 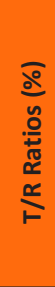 } & 278.51 & 247.18 & $286.81^{*}$ & 267.94 & 261.61 & 261.45 \\
\hline 125.7363 & & 330.32 & 293.16 & 340.17 & 317.78 & 310.27 & 310.09 \\
\hline 119.4939 & & 347.58 & 308.48 & 357.94 & 334.39 & 326.48 & 326.29 \\
\hline 130.2147 & & 318.96 & 283.08 & $328.47^{\#}$ & 306.86 & 299.60 & 299.43 \\
\hline 122.7268 & & 338.42 & 300.35 & 348.51 & 325.58 & 317.88 & 317.70 \\
\hline 131.7954 & & 315.13 & 279.68 & 324.53 & 303.18 & 296.01 & 295.84 \\
\hline \multicolumn{8}{|c|}{ Rank Order } \\
\hline & & 5 & 1 & $8^{*}$ & 4 & 3 & 2 \\
\hline & & 30 & 9 & 33 & 22 & 19 & 18 \\
\hline & & 34 & 17 & 36 & 31 & 28 & 27 \\
\hline & & 24 & 7 & $29^{\#}$ & 16 & 13 & 12 \\
\hline & & 32 & 14 & 35 & 26 & 23 & 21 \\
\hline & & 20 & 6 & 25 & 15 & 11 & 10 \\
\hline
\end{tabular}

*indicates $8^{\text {th }}$ rank; \#indicates $29^{\text {th }}$ rank.

T: Test (0.5\% Terbinafine film-forming solution); R: Reference (1\% Terbinafine film-forming solution); Cl: confidence interval. 
Table 3. T/R Ratio of Release Rates of Test (2.0\%) vs. Reference Formulation (1.0\%)

\begin{tabular}{|c|c|c|c|c|c|c|c|}
\hline \multirow{3}{*}{ Test Release Rate (Slope) } & \multicolumn{7}{|c|}{ Reference Release Rate (Slope) } \\
\hline & & 415.3325 & 368.6100 & 427.7148 & 399.5709 & 390.1243 & 389.8974 \\
\hline & \multicolumn{7}{|c|}{ T/R Ratios (\%) } \\
\hline 831.5171 & \multirow{6}{*}{ 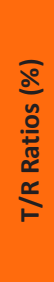 } & 49.95 & 44.33 & 51.44 & 48.05 & 46.92 & 46.89 \\
\hline 881.1857 & & 47.13 & 41.83 & $48.54^{\#}$ & 45.34 & $44.27 *$ & 44.25 \\
\hline 859.1792 & & 48.34 & 42.90 & 49.78 & 46.51 & 45.41 & 45.38 \\
\hline 808.8282 & & 51.35 & 45.57 & 52.88 & 49.40 & 48.23 & 48.21 \\
\hline 879.7174 & & 47.21 & 41.90 & 48.62 & 45.42 & 44.35 & 44.32 \\
\hline 890.4992 & & 46.64 & 41.39 & 48.03 & 44.87 & 43.81 & 43.78 \\
\hline \multicolumn{8}{|c|}{ Rank Order } \\
\hline & & 33 & 10 & 35 & 25 & 21 & 20 \\
\hline & & 22 & 2 & $29^{\#}$ & 13 & $8^{*}$ & 7 \\
\hline & & 28 & 4 & 32 & 18 & 15 & 14 \\
\hline & & 34 & 17 & 36 & 31 & 27 & 26 \\
\hline & & 23 & 3 & 30 & 16 & 11 & 9 \\
\hline & & 19 & 1 & 24 & 12 & 6 & 5 \\
\hline
\end{tabular}

*indicates $8^{\text {th }}$ rank; ${ }^{*}$ indicates $29^{\text {th }}$ rank.

T: Test (2.0\% Terbinafine film-forming solution); R: Reference (1\% Terbinafine film-forming solution); Cl: confidence interval.

Table 4. T/R Ratio of Release Rates of Test (1.0\%) vs. Reference Formulation (1.0\%)

\begin{tabular}{|c|c|c|c|c|c|c|c|}
\hline \multirow{3}{*}{ Test Release Rate (Slope) } & \multicolumn{7}{|c|}{ Reference Release Rate (Slope) } \\
\hline & & 402.3883 & 417.6389 & 389.6391 & 395.8690 & 384.7116 & 424.9194 \\
\hline & \multicolumn{7}{|c|}{ T/R Ratios (\%) } \\
\hline 415.3325 & \multirow{6}{*}{ 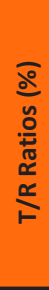 } & 103.22 & 99.45 & 106.59 & 104.92 & 107.96 & 97.74 \\
\hline 368.6100 & & 91.61 & 88.26 & 94.60 & 93.11 & 95.81 & 86.75 \\
\hline 427.7148 & & 106.29 & 102.41 & 109.77 & 108.04 & 111.18 & 100.66 \\
\hline 399.5709 & & 99.30 & 95.67 & 102.55 & 100.94 & $103.86^{\#}$ & 94.03 \\
\hline 390.1243 & & 96.95 & $93.41 *$ & 100.12 & 98.55 & 101.41 & 91.81 \\
\hline 389.8974 & & 96.90 & 93.36 & 100.07 & 98.49 & 101.35 & 91.76 \\
\hline \multicolumn{8}{|c|}{ Rank Order } \\
\hline & & 28 & 19 & 32 & 30 & 33 & 15 \\
\hline & & 3 & 2 & 10 & 6 & 12 & 1 \\
\hline & & 31 & 26 & 35 & 34 & 36 & 22 \\
\hline & & 18 & 11 & 27 & 23 & $29^{\#}$ & 9 \\
\hline & & 14 & $8^{*}$ & 21 & 17 & 25 & 5 \\
\hline & & 13 & 7 & 20 & 16 & 24 & 4 \\
\hline
\end{tabular}

*indicates $8^{\text {th }}$ rank; ${ }^{*}$ indicates $29^{\text {th }}$ rank.

T: Test (1\% Terbinafine film-forming solution); R: Reference (Lamisil Once, 1\% cutaneous solution); Cl: confidence interval.

\section{Statistical Evaluation via SUPAC-SS}

The $90 \% \mathrm{Cl}$ was calculated. As shown in Table 4, the 8th and 29th ranked values are $93.41 \%$ and $103.86 \%$, respectively. This indicates the $90 \% \mathrm{Cl}$ is within the limits of $75-133 \%$ in accordance with SUPAC-SS guidance.

\section{CONCLUSION}

For effective pharmacological action of any topical dosage forms, the formulation must reach the site of action and before that it must be released from the vehicle to penetrate the skin layers. For estimating the release rate from the topical formulations, the IVRT is a useful tool. During the drug development stage, IVRT can be used to finalize the formulation before clinical trials and to check the lot-to-lot consistency. The aim of this study was to establish a fast, accurate, and reproducible in vitro release method for determination of TBF from its topical film-forming solution formulations. In this study, drug release was consistent $\left(R^{2}>0.99\right)$ and release rates were reproducible $(\% \mathrm{CV}<15 \%)$. The percent cumulative 
release (recovery) after 6 hours of sampling was less than $30 \%$ of the applied formulation, which indicates the sink condition was maintained throughout the experiment. Release rates between test and reference formulations were compared statistically through a widely accepted $\mathrm{T} / \mathrm{R}$ ratio method, and results fell within $90 \% \mathrm{Cl}$ limits hence, confirming the product sameness.

The developed and validated IVRT and HPLC methods are suitable for drug release testing and routine testing of TBF cutaneous and film-forming solutions. Furthermore, this method can also be applied for determination of release rates of other TBF topical formulations.

\section{CONFLICT OF INTEREST}

The authors disclosed no conflicts of interest related to this article.

\section{REFERENCES}

1. Ryder, N. S. Terbinafine: mode of action and properties of the squalene epoxidase inhibition. Br. J. Dermatol. 1992, 126, 2-7. DOI: 10.1111/j.1365-2133.1992.tb00001.x.

2. Kanfer, I.; Rath S.; Purazi, P.; Mudyahoto, N. A. In Vitro Release Testing of Semi-Solid Dosage Forms. Dissolut. Technol. 2017, 24, 52-60. DOI: dx.doi.org/10.14227/DT240317P52.
3. Tran, T. T. D.; Tran, P. H. L. Controlled release film forming systems in drug delivery: the potential for efficient drug delivery. Pharmaceutics. 2019, 290, 1-16. DOI: 10.3390/ pharmaceutics 11060290.

4. Higuchi, T. Rate of release of medicaments from ointment bases containing drugs in suspension. J. Pharm. Sci. 1961, 50, 874-875. DOI: $10.1002 /$ jps.2600501018.

5. Kanakapura, B.; Penmatsa, V. K. Analytical methods for determination of terbinafine hydrochloride in pharmaceuticals and biological materials. J. Pharm. Anal. 2016, 6, 137-149. DOI: 10.1016/j. jpha.2016.01.003.

6. Nonsterile Semisolid Dosage Forms, Scale-Up and Postapproval Changes: Chemistry, Manufacturing, and Controls; In Vitro Release Testing and In Vivo Bioequivalence Documentation; Guidance for Industry; U.S. Department of Health and Human Services, Food and Drug Administration, Center for Drug Evaluation and Research (CDER), U.S. Government Printing Office: Washington, DC, 1997.

7. Tiffner, K. I.; Kanfer, I.; Augustin, T.; Raml, R.; Raney, S. G.; Sinner, F. A comprehensive approach to qualify and validate the essential parameters of an in vitro release test (IVRT) method for acyclovir cream, 5\%. Int. J. Pharm. 2018, 535, 217-227. DOI: 10.1016/j. ijpharm.2017.09.049. 